\title{
A Simple Scheme to Improve the Throughput of Small Multi-hop Wireless Networks
}

\author{
S. D. Gunashekar ${ }^{*}$, A. Das ${ }^{\S}$, T. Erlebach ${ }^{*}$, and E. M. Warrington ${ }^{\dagger}$ \\ "Department of Computer Science, University of Leicester, Leicester, United Kingdom \\ $\S_{\text {TTE Systems Ltd, Leicester, United Kingdom }}$ \\ ${ }^{\dagger}$ Department of Engineering, University of Leicester, Leicester, United Kingdom \\ $\{$ sdg10, ad108, te17, emw\}@1e.ac.uk
}

\begin{abstract}
This paper proposes a simple scheme to optimise the end-to-end user throughput of small, multi-hop wireless networks. The scheme which involves the introduction of small delays between successive packet transmissions at the source node has been tested on an indoor wireless mesh testbed for uni-directional flows of data traffic with nodes that use Chirp Spread Spectrum technology. Results from a series of novel experiments confirm the effectiveness of the technique to significantly improve the throughput of multihop wireless networks.
\end{abstract}

Keywords-mesh networks; multi-hop wireless networks; wireless testbed; throughput optimisation; Chirp Spread Spectrum

\section{INTRODUCTION}

Over the last few years, wireless mesh networks have become a feasible means of communication as a consequence of their cost-effectiveness and the relative ease with which they can be deployed. There is no centralized infrastructure controlling these multi-hop networks and their inherent "self-healing" ability improves the overall reliability of the system [1]. While the majority of the research in this field focusses on either simulation studies (using, for example, $n s-2$ [2] or OPNET [3]) or theoretical analyses, in order to provide a better understanding of mesh networks, there has been a small but growing trend to implement wireless mesh testbeds [1, 4-7].

The end-to-end throughput of the system is a vital parameter that needs to be considered in the design of multi-hop wireless networks. An attempt has been made to develop a scheme to improve the resultant throughput of wireless multi-hop "chain" networks with varying numbers of nodes. The scheme has been devised to operate in the specific context of multi-hop networks employing nodes with Chirp Spread Spectrum (CSS) technology, a technique that facilitates robust wireless communications with low power consumption [8, 9]. As far as the authors are aware, this is the first time that the throughput optimisation of CSS-based multi-hop networks has been investigated.

The proposed throughput optimisation scheme is discussed in Section II. The wireless testbed used in the experiments is described in Section III. In Section IV, results are presented from a series of experiments carried out to evaluate the effectiveness of the scheme. Finally, some concluding remarks are provided in Section V.

\section{DESCRIPTION OF THE OPTIMISATION SCHEME}

The scheme involves the introduction of a delay at the transmitting node of a linear multi-hop network between successive packet transmissions (i.e., the scheme follows the pattern: transmit packet, wait, transmit packet, wait, transmit packet, wait ...). The delay essentially controls the rate at which packets are being generated at the transmitter. Therefore, instead of the intermediate node(s) being continuously hit with packets - a number of which get dropped under normal circumstances - an optimal delay will ensure that each packet travels a sufficient number of hops away from the source, before the next packet is transmitted.

\section{EXPERIMENTAL SET-UP}

A wireless testbed employing several Nanotron nanoLOC boards [9] has been constructed. Each board operates in the industrial, scientific and medical frequency band $(2.4 \mathrm{GHz})$ and has been interfaced to a $P C / 104$ based single board computer. An individual node in the testbed consists of a compact, waterproof and battery-powered radio-PC unit which can be easily deployed in the field. The transceiver chip on the board is capable of supporting four primary logical transmit channels, each of which is defined by specific attributes such as data rate, centre frequency and bandwidth. In all the measurements presented in this paper, the Chirp Spread Spectrum logical transmit channel, with a data rate of $1.0 \mathrm{Mbps}$, centre frequency of $2.44175 \mathrm{GHz}$ and bandwidth of $80 \mathrm{MHz}$, was employed. Further specifications about the testbed can be found in [10].

The performance of multi-hop networks that consist of a "chain" or "string" of static wireless nodes has been examined in this paper. Each node was given a unique medium access control (MAC) address and packets were delivered from the source node in the chain to the destination node by using a static routing strategy. All the experiments reported in this paper were performed in a university laboratory setting which provided an environment that was prone to multipath and scattering. Additionally, it must be noted that in some of the experiments, there were significant levels of interference from the university's WiFi network. Results from several 
point-to-point single-hop experiments and preliminary multi-hop experiments involving the Nanotron nodes have previously been presented in [10].

\section{EXPERIMENTAL RESULTS}

\section{A. Typical Throughput Measuements}

Before examining the testbed results involving the delay scheme, for the purpose of comparison, it is worth presenting some typical throughput measurements that were carried out without the use of any throughput optimisation scheme. Experiments were performed with linear networks with up to 10 Nanotron nodes. A transmit power of $-34.47 \mathrm{dBm}$ was used at all the nodes which were placed at heights of $1.5 \mathrm{~m}$ above floor level. Because of space constraints in the laboratory, the internode separation was fixed at approximately $0.80 \mathrm{~m}$. The packet size was set to 128 bytes.

A graph depicting the mean (over a 2-minute data acquisition period in which one throughput reading was logged every second) net throughputs for the various chain lengths is shown in Figure 1. The typical curve illustrating throughput degradation with increasing number of nodes is observed. The reasons for this behaviour have been discussed in detail in [10] and [11]. From the maximum end-to-end throughput ( $308 \mathrm{kbps})$ corresponding to the 2-node network, the throughput for the 3-node chain drops sharply to approximately $71 \mathrm{kbps}$. After further dropping to around $27 \mathrm{kbps}, 18 \mathrm{kbps}$, and $14 \mathrm{kbps}$, respectively, for the 4-node, 5-node and 6-node chains, the mean throughput stabilises at approximately 10-11 kbps for networks with 7 or more nodes.

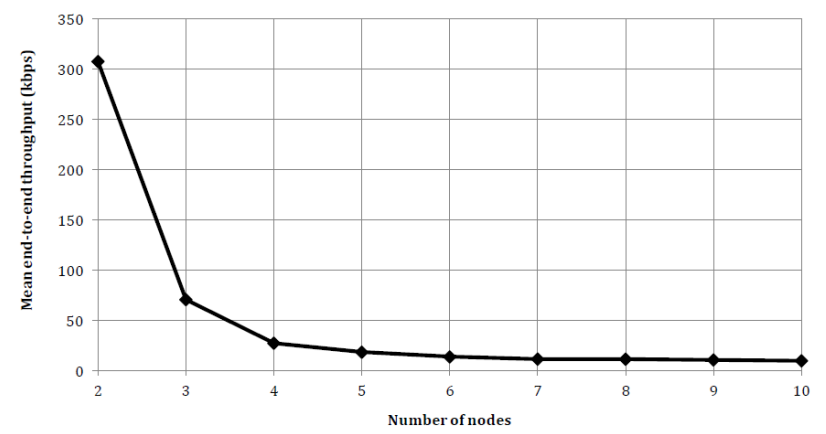

Figure 1. For various chain lengths, mean throughputs measured at the receiving nodes of the respective linear chains (transmit power: $-34.47 \mathrm{dBm}$; inter-node separation: $0.80 \mathrm{~m}$; height of nodes: $1.5 \mathrm{~m}$ above floor level).

\section{B. Implementation of the Delay Scheme}

The delay scheme was applied to linear multi-hop networks with uni-directional data traffic flows. Specifically, experiments were performed to verify the performance of 2-node, 3-node, 4-node and 5-node CSSbased networks. The inter-node separation was fixed at approximately $1.5 \mathrm{~m}$ and each node was placed at a height of around $1.5 \mathrm{~m}$ above ground level. The packet size was set to 128 bytes and a constant transmit power of $-34.47 \mathrm{dBm}$ was used at every node in the network.
For each experimental configuration, a range of delay values were tested to determine an optimal value. Furthermore, 2-3 "no delay" or "0 ms" measurements were also carried out through the course of each measurement to verify whether the propagation conditions had changed. These were generally performed - for each configuration - at the beginning and end of the experimental run, and in some cases, midway through the measurements. It is important to mention that during testing it was determined that the experimentally measured delay values were in fact a few milliseconds (3$10 \mathrm{~ms}$ ) greater than the delay value specified in the source code. This additional delay has been specified as " $\Delta$ " in the text. The delay values hereafter refer to the values specified in the code - the "actual" delay values between consecutive data packets will be slightly higher.

2 nodes: For a simple single transmitter-single receiver link, insertion of a delay between successive packet transmissions should have a negative impact on the throughput performance of the system and reduce the throughput rather than increase it. With no intermediate nodes to pass through, the rate at which packets are generated at the transmitting node simply decreases with the addition of a delay value. Fewer packets are therefore received at the destination node per unit time, thus resulting in a reduction in throughput. This is exactly what is observed from the results of the 2-node delay measurements presented in Figure 2 in which mean throughput has been plotted as a function of the delay value introduced. As the length of the delay is increased, there is a subsequent drop in the measured throughput. Without any delay, the mean end-to-end net throughput is approximately $291 \mathrm{kbps}$. However, a delay value of, say $(10+\Delta) \mathrm{ms}$, results in a drastic reduction in the throughput to around $80 \mathrm{kbps}$.

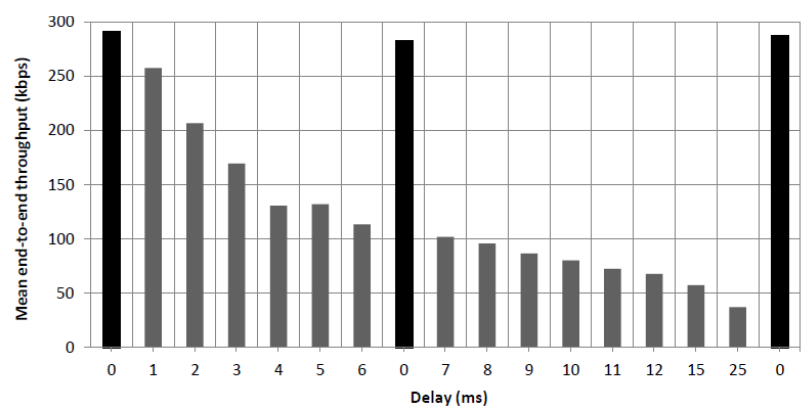

Figure 2. Mean throughput values recorded at the receiving node of a 2node linear chain for different delay values inserted between successive packet transmissions at the transmitting node (transmit power: $-34.47 \mathrm{dBm}$; inter-node separation: $1.5 \mathrm{~m}$; height of nodes: $1.5 \mathrm{~m}$ above floor level)

It may be noted that the sequence in which the delay values have been presented on the $\mathrm{x}$-axis in Figure 2 (as well as in all the remaining figures in this paper) also corresponds to the order in which the particular delay value measurement was performed. So, in Figure 2, the $(2+\Delta)$ ms delay measurement was performed immediately after the $(1+\Delta)$ ms delay measurement and just before the $(3+\Delta)$ ms delay measurement, while the 
$0 \mathrm{~ms}$ measurements were the first, eighth and last measurements.

3 nodes: The results for a 3-node linear chain are shown in Figure 3. The reasonable similarity in the "zero delay" throughput values (shown in black) obtained at the start ( $\sim 73 \mathrm{kbps})$ and end ( $68 \mathrm{kbps})$ of the measurements provide an indication that the propagation conditions did not vary too significantly during the course of the experiments. A number of delay values between $(4+\Delta) \mathrm{ms}$ and $(10+\Delta) \mathrm{ms}$ result in throughput gains. The optimal delay value is $(5+\Delta)$ ms which results in the maximum throughput gain: the mean throughput increases by approximately $47 \%$ from $73 \mathrm{kbps}$ (the "zero delay" mean throughput) to around $107 \mathrm{kbps}$. The delay value of $(5+\Delta)$ ms roughly corresponds to the amount of time one would expect a packet to take to reach the destination.

At higher delay values, the packet sending rate of the source node decreases significantly. Under these circumstances, the probability of packet collisions along the chain decreases (each packet now has more than sufficient time to reach the destination node via the intermediate hops). With a delay of $(15+\Delta) \mathrm{ms}$, for instance, there are hardly any lost packets recorded at the destination node (and the intermediate node) of the 3-node chain; however, with such a large delay in the system, the transmitter is sending out significantly fewer packets every second, with the consequence that fewer packets are received at the destination node. Thus, with the insertion of higher-than-optimal delay values, although there may be a reduction in the end-to-end throughput, the system attains a more steady state with significantly fewer dropped packets along the chain.

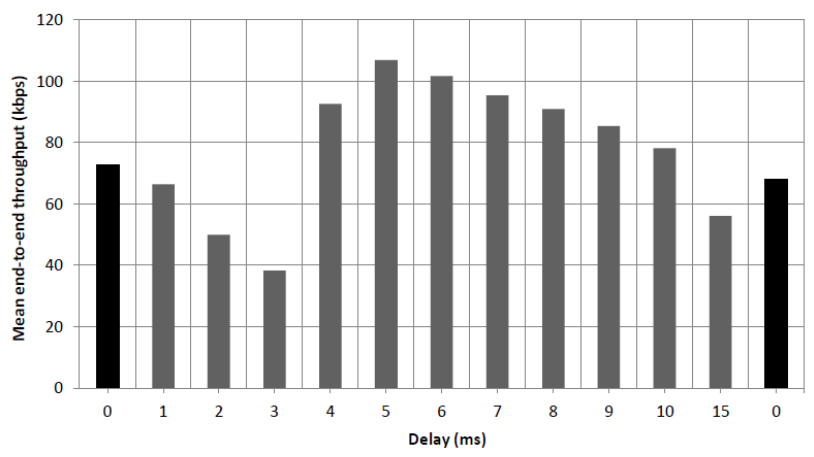

Figure 3. Mean throughput values recorded at the receiving node of a 3node linear chain for different delay values inserted between successive packet transmissions at the transmitting node (transmit power: $-34.47 \mathrm{dBm}$; inter-node separation: $1.5 \mathrm{~m}$; height of nodes: $1.5 \mathrm{~m}$ above floor level)

4 nodes: The results of the delay measurements for the 4-node chain are presented in Figure 4. Once again, "zero delay" throughputs were recorded at the beginning and end of the measurement resulting in mean end-to-end throughputs of approximately $56 \mathrm{kbps}$ and $62 \mathrm{kbps}$, respectively. Delay values between $(4+\Delta) \mathrm{ms}$ and $(12+\Delta) \mathrm{ms}$ result in throughput gains. With a longer chain, it would be expected that a greater delay value would result in the maximum throughput gain. However, as in the case of the 3-node chain, the optimal delay value is $(5+\Delta) \mathrm{ms}$. With this delay at the source node, the endto-end throughput improves by approximately $45 \%$ from about $56 \mathrm{kbps}$ to around $82 \mathrm{kbps}$. A second throughput peak also occurs at $(9+\Delta) \mathrm{ms}$. Midway through the measurements, it was noticed that there was an increase in the netbook activity in the adjoining areas of the laboratory that resulted in disruption of the wireless multihop network. During this period, there was more fluctuation in the instantaneous throughputs as evidenced by the unusually large standard deviation values noted in some of the measurements. For this reason, the delay measurements for the 4-node chain (and 3-node and 5node chains) were repeated (these additional measurements are presented at the end of this section after the 5-node measurements).

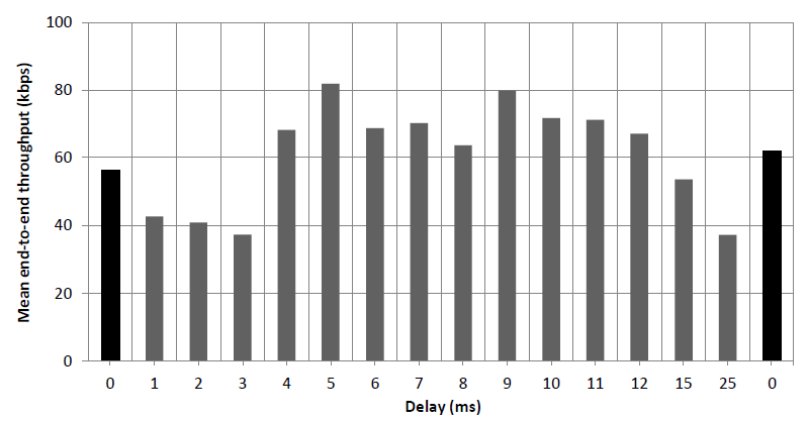

Figure 4. Mean throughput values recorded at the receiving node of a 4node linear chain for different delay values inserted between successive packet transmissions at the transmitting node (transmit power: $-34.47 \mathrm{dBm}$; inter-node separation: $1.5 \mathrm{~m}$; height of nodes: $1.5 \mathrm{~m}$ above floor level)

5 nodes: The throughput versus delay measurements for the 5-node chain is shown in Figure 5. As expected, longer delay values between approximately $(7+\Delta) \mathrm{ms}$ and $(15+\Delta) \mathrm{ms}$ result in throughput improvements. The maximum throughput gain is attained when a delay of $(8+\Delta) \mathrm{ms}$ is applied at the transmitter: a mean throughput of around $45 \mathrm{kbps}$ is achieved. The three "zero delay" measurements performed at the beginning of the experiment, midway through the experiment and at the end of the experiment result in throughput values of $38 \mathrm{kbps}, 26 \mathrm{kbps}$ and $25 \mathrm{kbps}$, respectively, indicating variable propagation conditions over the course of the entire measurement period. Using the initial "zero delay" measurement $(38 \mathrm{kbps})$ as the base measurement, the $(8+\Delta) \mathrm{ms}$ delay results in a throughput gain of approximately $19 \%$. If, however, the "zero delay" measurement in the middle of the experiment $(26 \mathrm{kbps})$ is utilised as the base measurement, the $(8+\Delta)$ ms delay results in a very substantial throughput gain of $70 \%$ (this is perhaps more accurate as these "zero delay" measurements are closer in time to the $(8+\Delta)$ ms delay measurements). As in the case of the 3-node and 4-node experiments, even though the higher-than-optimal delay values result in progressively lower throughputs, there are significantly fewer lost packets across each hop of the chain as a result of the source node generating a smaller number of packets per unit time. 


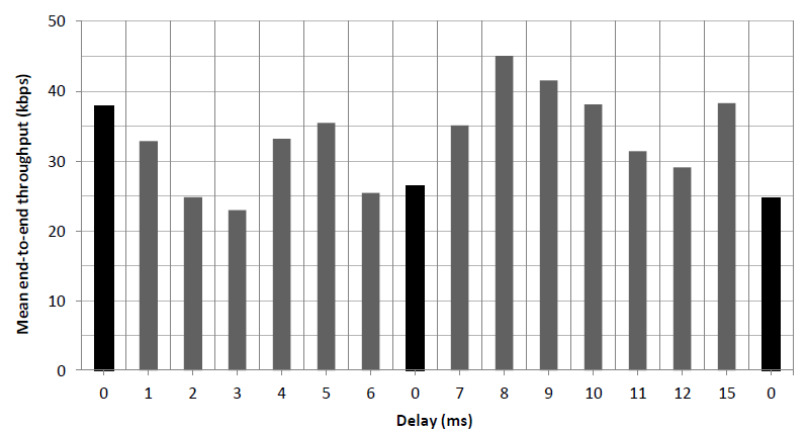

Figure 5. Mean throughput values recorded at the receiving node of a 5node linear chain for different delay values inserted between successive packet transmissions at the transmitting node (transmit power: $-34.47 \mathrm{dBm}$; inter-node separation: $1.5 \mathrm{~m}$; height of nodes: $1.5 \mathrm{~m}$ above floor level)

Repeat measurements: The results from the "repeat measurements" for the 3-node, 4-node and 5-node chains are presented in Figures 6, 7 and 8 . Most of the experimental parameters were identical to those used in the previous measurements (only the inter-node separations and the heights of the nodes varied slightly). Unfortunately, however, and particularly with respect to the 3-node and 5-node chains, interference was again observed from the university's WiFi network (this is indicated by the variable "zero delay" measurements conducted at the beginning, midway through and end of the experiment). Less interference was experienced at the time of conducting the 4-node experiments; consequently the results from these measurements show relatively less variation compared to the previous set of measurements.

In all three experiments, a collection of delay values introduced at the respective transmitting nodes produced throughput gains. For the 3-node chain, this delay range is approximately $(5+\Delta) \mathrm{ms}$ to $(15+\Delta) \mathrm{ms}$. 4-node chains exhibit throughput enhancements when delays in the range of $(4+\Delta) \mathrm{ms}$ to $(20+\Delta) \mathrm{ms}$ are applied to the source node. Finally, with respect to the 5-node chain, delays between approximately $(4+\Delta) \mathrm{ms}$ and $(40+\Delta) \mathrm{ms}$ result in throughput enhancements.

As the chain length increases, progressively higher delay values are required to optimise the end-to-end throughput: these were $(7+\Delta) \mathrm{ms}$ for the 3-node chain (resulting in a throughput improvement of almost 59\%), $(8+\Delta) \mathrm{ms}$ for the 4-node chain (resulting in a throughput improvement of around $84 \%)$, and $(14+\Delta) \mathrm{ms}$ for the 5-node chain (resulting in a throughput improvement in excess of $100 \%$ ). Thus, by merely using a simple scheme in which a delay is introduced between successive packet transmissions to control the rate at which packets are generated at the source node, significant improvements in multi-hop throughput are possible. The precise value of the optimal delay, however, is most likely to vary with the experimental conditions.

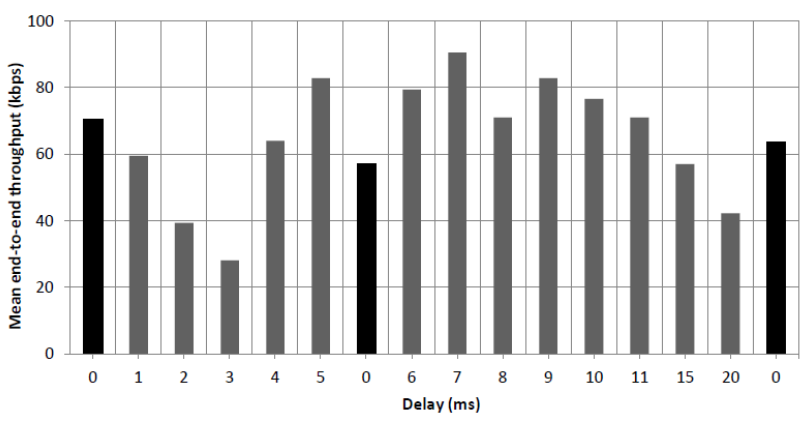

Figure 6. Mean throughput values recorded at the receiving node of a 3node linear chain for different delay values inserted between successive packet transmissions at the transmitting node (transmit power: $-34.47 \mathrm{dBm}$; inter-node separation: $1.15 \mathrm{~m}$; height of nodes: $1.4 \mathrm{~m}$ above floor level)

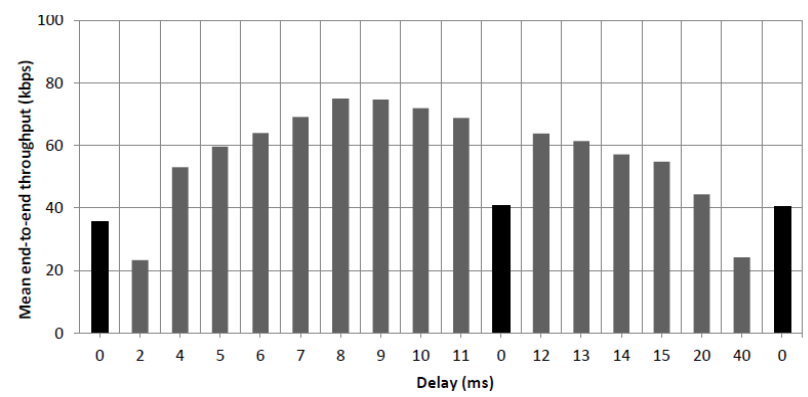

Figure 7. Mean throughput values recorded at the receiving node of a 4node linear chain for different delay values inserted between successive packet transmissions at the transmitting node (transmit power: $-34.47 \mathrm{dBm}$; inter-node separation: $1.15 \mathrm{~m}$; height of nodes: $1.4 \mathrm{~m}$ above floor level)

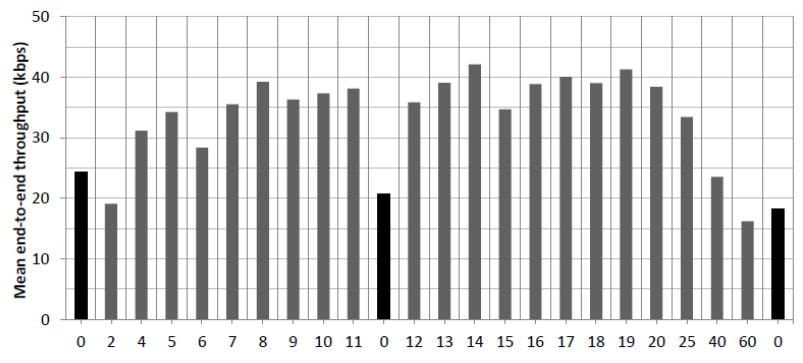
Delay (ms)

Figure 8. Mean throughput values recorded at the receiving node of a 5node linear chain for different delay values inserted between successive packet transmissions at the transmitting node (transmit power: $-34.47 \mathrm{dBm}$; inter-node separation: $1.15 \mathrm{~m}$; height of nodes: $1.4 \mathrm{~m}$ above floor level)

\section{CONCLUDING REMARKS}

In order to improve the end-to-end throughput of wireless multi-hop networks with a small number of nodes, a simple optimisation scheme has been devised in which a delay between successive packet transmissions is introduced at the source node of the network. The scheme was tested using Chirp Spread Spectrum based, linear chain, multi-hop networks with uni-directional data traffic flows. In all the configurations, the scheme was successfully applied to yield significant enhancements in the end-to-end throughput. In each case, a range of delay values produced throughput gains, with longer chains requiring progressively greater delays. For example, for a 3 -node chain, in the first set of experiments, delays in the range $(4+\Delta) \mathrm{ms}$ to $(10+\Delta) \mathrm{ms}$ resulted in throughput improvements, with $(5+\Delta) \mathrm{ms}$ being the optimal delay 
value which produced a $47 \%$ enhancement in throughput. With respect to a 4-node chain, in the repeat experiments, a collection of delay values in the range $(4+\Delta) \mathrm{ms}$ to $(20+\Delta)$ ms produced throughput gains, with $(8+\Delta) \mathrm{ms}$ resulting in a mean throughput that was approximately $84 \%$ higher than the "zero delay" throughput in one set of measurements. Finally, with the inclusion of delays in the range $(4+\Delta) \mathrm{ms}$ to $(40+\Delta) \mathrm{ms}$, significant throughput improvements were observed with 5-node linear chain networks in the repeat experiments.

With regard to future work, the delay scheme demonstrated in this paper will be applied to multi-hop networks consisting of bi-directional data flows (preliminary investigations have indicated that there is scope for significantly improving the 2-way traffic throughput). Additionally, measurements involving the delay scheme will be performed with static linear networks having longer chain lengths, as well as with multi-hop networks with more random topologies. Experiments will be carried out in both indoor and outdoor environments. Finally, it would also be useful to develop an analytical model to validate the throughput optimisation scheme.

\section{REFERENCES}

[1] I. F. Akyildiz, X. Wang, and W. Wang, "Wireless Mesh Networks: a Survey," Computer Networks, 47(4), pp. 445-487, 2005.

[2] $n s-2$ website. http://www.isi.edu/nsnam/ns/. Accessed 28 November 2011.

[3] OPNET website. http://www.opnet.com/index.html. Accessed 28 November 2011.

[4] D. Aguayo, J. Bicket, S. Biswas, G. Judd, and R. Morris, "Linklevel Measurements from an $802.11 \mathrm{~b}$ Mesh Network," Proceedings of the 2004 conference on applications, technologies, architectures, and protocols for computer communications (SIGCOMM 2004), Portland, Oregon, USA, 2004.

[5] W. Kiess and M. Mauve, "A survey on real-world implementations of mobile ad-hoc networks," Ad Hoc Networks, 5(3), pp. 324-339, 2007, DOI:10.1016/j.adhoc.2005.12.003.

[6] S. M. ElRakabawy, S. Frohn, and C. Lindemann, "A Scalable Dual-Radio Wireless Testbed for Emulating Mesh Networks," Wireless Networks, 16(8), pp. 2191-2207, 2010, DOI:10.1007/s11276-010-0253-3.

[7] B. Blywis M. Günes, F. Juraschek, and J.H. Schiller, "Trends, Advances, and Challenges in Testbed-based Wireless Mesh Network Research," Mobile Networks and Applications, 15(3), pp. 315-329, 2010, DOI:10.1007/s11036-010-0227-9.

[8] Nanotron Technologies GmbH, "nanoNET Chirp Based Wireless Networks", White Paper, 2007,

http://www.nanotron.com/EN/pdf/WP_CSS.pdf. Accessed $30 / 05 / 2012$.

[9] Nanotron Technologies GmbH, "nanoLOC Development Kit 3.0", Factsheet, http://www.nanotron.com/EN/pdf/Factsheet nanoLOCDev-Kit.pdf. Accessed 30/05/2012.

[10] S. D. Gunashekar, A. Das, T. Erlebach, and E. M. Warrington, "Wireless multi-hop throughput: preliminary testbed measurements," Proceedings of the Loughborough Antennas and Propagation Conference 2011 (LAPC 2011), Loughborough, UK, 2011, DOI:10.1109/LAPC.2011.6114121.

[11] S. D. Gunashekar, A. Das, T. Erlebach, and E. M. Warrington, "Wireless multi-hop throughput: preliminary results from a simulation-based study," Proceedings of the Loughborough Antennas and Propagation Conference 2011 (LAPC 2011), Loughborough, UK, 2011, DOI:10.1109/LAPC.2011.6114122. 